\title{
Resolving Cosmological Singularities
}

\section{Ali H. Chamseddine ${ }^{1,2}$, Viatcheslav Mukhanov ${ }^{3,4,5}$}

${ }^{1}$ Physics Department, American University of Beirut, Lebanon

${ }^{2}$ I.H.E.S. F-91440 Bures-sur-Yvette, France

Niels Bohr International Academy, Niels Bohr Institute, Blegdamsvej 17, DK-2100 Copenhagen, Denmark

${ }^{4}$ Theoretical Physics, Ludwig Maxmillians University, Theresienstr. 37, 80333 Munich, Germany

${ }^{5}$ MPI for Physics, Foehringer Ring, 6, 80850, Munich, Germany

\begin{abstract}
We find a simple modification of the longitudinal mode in General Relativity which incorporates the idea of limiting curvature. In this case the singularities in contracting Friedmann and Kasner universes are avoided, and instead, the universe has a regular bounce which takes place during the time inversely proportional to the square root of the limiting curvature. Away from the bounce, corrections to General Relativity are negligible. In addition the non-singluar modification of General Relativity delivers for free a realistic candidate for Dark Matter.
\end{abstract}




\section{Introduction}

One of the long standing unsolved problem in General Relativity is the problem of spacelike singularities. In fact, assuming that General Relativity is universally valid and imposing rather general conditions on the state of matter, Hawking and Penrose have proven that space-times describing such as, for instance, Friedmann and Kasner universes and black holes, are geodesically incomplete [1]. Gravitational collapse leads to singularities where the curvature invariants grow and become infinite. It is well known that when the curvature approaches the Planckian value quantum effects become extremely important and Einstein theory must be modified. This is the reason for the common belief that a nonperturbative quantum gravity must finally resolve the singularities. In fact, near the singularity, vacuum polarization induces higher order curvature corrections to the Einstein action and particle production becomes very important [2]. These effects must completely modify General Relativity when the Planck curvature is approached. Unfortunately, there is no reliable way to calculate constructively all these effects nonperturbatively and therefore the "quantum resolution" of singularities remains rather obscure, and at the moment it is not more than wishful thinking. In fact progress along this line of research has been relatively modest in spite of the enormous efforts devoted to the problem.

There is a different "non-quantum" approach to resolving the singularities. In fact, one can imagine that at high curvatures, classical General Relativity is modified to incorporate the idea of limiting density/curvature [3]. If this limiting curvature is at least few orders of magnitude below the Planckian value, then one can safely ignore nonperturbative quantum gravity effects and can entirely rely on the classical theory used in investigating the singularities. On the other hand, if the limiting curvature is large enough and the theory reduces to General Relativity at low curvature, it will not be in contradiction with experiment and therefore such a theory will be fully legitimate. Once again the progress in solving the singularity problem using this approach is rather modest. In the papers [4] [5] it was suggested to modify Einstein's theory by adding some complicated combination of higher order curvature invariants which allowed obtaining a non-singluar homogeneous isotropic universe. However, it is totally unclear how to extend the proposal in these papers, which is rather messy and complicated, to the cases of anisotropic singularities and the singularity "inside" the black hole.

In this letter we shall consider a minimal simple modification of Einstein's 
theory, where the singularities are avoided at the classical level in Friedmann and Kasner 8] universes irrespective of the matter which fills the universe. In an accompanying paper we will show that black holes in this theory are also nonsingluar. This opens the possibility to have a theory of gravity where singularities are absent in general. To construct such a theory we will introduce the constrained scalar field $\phi$, which satisfies

$$
g^{\mu \nu} \partial_{\mu} \phi \partial_{\nu} \phi=1
$$

As it was shown in [6], this constrained scalar field is not dynamical by itself, but induces mimetic dark matter in Einstein theory making the longitudinal degree of freedom of the gravitational field dynamical. Notice that its emergence can, for example, be justified in noncommutative geometries as a consequence of quantization of thee-dimensional volume [9]. In a previous paper [7], by allowing a potential term $V(\phi)$, we were able to find bouncing nonsingular solutions for isotropic, homogeneous universe, but even in this case, the most general solutions remain singular. In addition to this drawback, the term $V(\phi)$ explicitly spoils time translational invariance because in the synchronous coordinate system the generic solution of (1) is $\phi=t+$ constant. We have also found that the potential term does not help in avoiding the singularity in Kasner universe and inside the black hole. Therefore we will assume here the absence of a potential term by requiring invariance with respect to the shift symmetry $\phi \rightarrow \phi+$ constant, and instead add to the Einstein action a function $f(\square \phi)$, which is invariant. The appearance of such function $f$ can be easily justified in the spectral action approach of noncommutative geometry [10] [11. Clearly we cannot derive it in nonperturbative way, but we can try just to find a theory where this function allows to resolve singularities in General Relativity. In particular, we will show that there exist Born-Infeld type of actions with

$$
f(\square \phi)=1-\sqrt{1-\frac{(\square \phi)^{2}}{\varepsilon_{m}}}+\ldots .
$$

and for which singularities are resolved, that is, the contracting universes bounce at the limiting curvature and all curvature invariants always remains regular and bounded by the values, characterized by $\varepsilon_{m}$. Once again we would like to stress that introducing $\square \phi$ does not add to the system any new dynamical scalar fields and new degrees of freedom. The "field $\phi$ " always remains constrained by (1) and in the synchronous coordinate system it just "serves" 
as time, making the longitudinal degree of freedom of the gravitational field to be dynamical. Thus, this theory must be viewed as a modification of General Relativity in the longitudinal sector. Because the longitudinal gravitational field induced by matter via the constraint has a "negative energy" by itself, it is not surprising that the proposed modification of Einstein's theory violates the conditions needed in the proof of singularity theorems and hence these singularities can be avoided. Although noncommutative geometry offers a very strong support for the model considered here [10], we will not require, or use any information or methods from that framework. Instead our purpose is to propose nonsingular classical modification of General Relativity irrespective of its justification from the point of view of so called "fundamental theory", which is not known at present.

\section{The theory}

Let us consider the theory described by the action

$$
S=\int\left(-\frac{1}{2} R+\lambda\left(g^{\mu \nu} \partial_{\mu} \phi \partial_{\nu} \phi-1\right)+f(\chi)+L_{m}\right) \sqrt{-g} d^{4} x,
$$

where $\chi=\square \phi, \lambda$ is a Lagrange multiplier, $L_{m}$ is the Lagrangian of the usual matter and we set $8 \pi G=1$. The mimetic scalar field $\phi$ satisfies the constraint [6] [7]

$$
g^{\mu \nu} \partial_{\mu} \phi \partial_{\nu} \phi=1
$$

and therefore the term $f(\chi)$ does not lead to the appearance of higher derivatives and ghost degrees of freedom. Variation of action (2) with respect to the metric gives the following equations

$$
G_{\mu \nu}=R_{\mu \nu}-\frac{1}{2} g_{\mu \nu} R=\tilde{T}_{\mu \nu}+T_{\mu \nu}
$$

where $T_{\mu \nu}$ is the energy-momentum tensor of the usual matter and

$$
\tilde{T}_{\mu \nu}=2 \lambda \partial_{\mu} \phi \partial_{\nu} \phi+g_{\mu \nu}\left(\chi f^{\prime}-f+g^{\rho \sigma} \partial_{\rho} f^{\prime} \partial_{\sigma} \phi\right)-\left(\partial_{\mu} f^{\prime} \partial_{\nu} \phi+\partial_{\nu} f^{\prime} \partial_{\mu} \phi\right),
$$

with $f^{\prime}=d f / d \chi$, describes the extra contribution to Einstein equations due to the $\phi$ dependent terms. In the synchronous coordinate system the metric takes the form [8]

$$
d s^{2}=d t^{2}-\gamma_{i k}\left(t, x^{l}\right) d x^{i} d x^{k}
$$


and if no coordinate singularities arise, the most general solution of constraint equation (3) is [8]

$$
\phi= \pm t+A,
$$

where $A$ is a constant of integration. In this coordinate system, the coordinate independent invariant $\chi=\square \phi$ becomes

$$
\chi=\square \phi=\frac{1}{\sqrt{-g}} \frac{\partial}{\partial x^{\mu}}\left(\sqrt{-g} g^{\mu \nu} \frac{\partial \phi}{\partial x^{\nu}}\right)=\frac{\dot{\gamma}}{2 \gamma},
$$

with $\gamma=\operatorname{det} \gamma_{i k}$ and dot denotes time derivative. In this paper we will consider flat Friedmann and Kasner universes, where the metric (6) depends only on time $t$, that is, $\gamma_{i k}=\gamma_{i k}(t)$. In this case the nonvanishing components of the curvature are 8]

$$
R_{0}^{0}=-\frac{1}{2} \dot{\varkappa}-\frac{1}{4} \varkappa_{i}^{k} \varkappa_{k}^{i}, \quad R_{k}^{i}=-\frac{1}{2 \sqrt{\gamma}} \frac{d\left(\sqrt{\gamma} \varkappa_{k}^{i}\right)}{d t}
$$

where $\varkappa_{k}^{i}=\gamma^{i m} \dot{\gamma}_{m k}, \varkappa=\varkappa_{i}^{i}=\dot{\gamma} / \gamma$. The components of mimetic matter contribution to the Einstein's equations are

$$
\begin{aligned}
& \tilde{T}_{0}^{0}=2 \lambda+\chi f^{\prime}-f-\dot{\chi} f^{\prime \prime}, \\
& \tilde{T}_{k}^{i}=\left(\chi f^{\prime}-f+\dot{\chi} f^{\prime \prime}\right) \delta_{k}^{i},
\end{aligned}
$$

The equation

$$
R_{0}^{0}-\frac{1}{2} R=\tilde{T}_{0}^{0}+T_{0}^{0}
$$

then takes the form

$$
\frac{1}{8}\left(\varkappa^{2}-\varkappa_{i}^{k} \varkappa_{k}^{i}\right)=2 \lambda+\chi f^{\prime}-f-\dot{\chi} f^{\prime \prime}+T_{0}^{0},
$$

The space-space equation

$$
R_{k}^{i}=\tilde{T}_{k}^{i}-\frac{1}{2} \tilde{T} \delta_{k}^{i}+T_{k}^{i}-\frac{1}{2} T \delta_{k}^{i},
$$

where $\tilde{T}=\tilde{T}_{\alpha}^{\alpha}$ and $T=T_{\alpha}^{\alpha}$, gives

$$
\frac{1}{2 \sqrt{\gamma}} \frac{\partial\left(\sqrt{\gamma} \varkappa_{k}^{i}\right)}{\partial t}=\left(\lambda+\chi f^{\prime}-f\right) \delta_{k}^{i}-T_{k}^{i}+\frac{1}{2} T \delta_{k}^{i} .
$$


To determine the Lagrange multiplier $\lambda$ it is convenient to integrate the equation obtained by variation of the action with respect to $\phi$ :

$$
\frac{1}{\sqrt{\gamma}} \partial_{0}(2 \sqrt{\gamma} \lambda)=\square f^{\prime}=\frac{1}{\sqrt{\gamma}} \partial_{0}\left(\sqrt{\gamma} f^{\prime \prime} \dot{\chi}\right),
$$

from which it follows that

$$
\lambda=\frac{C}{2 \sqrt{\gamma}}+\frac{1}{2} f^{\prime \prime} \dot{\chi}
$$

where $C$ is a constant of integration corresponding to mimetic cold matter.

Let us assume that for the usual matter $T_{k}^{i}=-p \delta_{k}^{i}$. This assumption is valid for all models we consider below and it is generic enough to understand how matter can influence singularities in case of general space-times. In this case, by subtracting from equation (14) one third of its trace, we obtain

$$
\frac{\partial}{\partial t}\left(\sqrt{\gamma}\left(\varkappa_{k}^{i}-\frac{1}{3} \varkappa \delta_{k}^{i}\right)\right)=0,
$$

and therefore

$$
\varkappa_{k}^{i}=\frac{1}{3} \varkappa \delta_{k}^{i}+\frac{\lambda_{k}^{i}}{\sqrt{\gamma}}
$$

where $\lambda_{k}^{i}$ are constants of integration which satisfy $\lambda_{i}^{i}=0$. Taking into account that $\varkappa=2 \chi=\dot{\gamma} / \gamma$ and substituting expression (18) together with (16) into (12) we arrive at

$$
\frac{1}{3} \chi^{2}+f-\chi f^{\prime}=\frac{\lambda_{k}^{i} \lambda_{i}^{k}}{8 \gamma}+\frac{C}{\sqrt{\gamma}}+T_{0}^{0},
$$

which is a first order differential equation for $\gamma$. Solving this equation and substituting the result in (18) we can determine all components of the metric.

There exists a large class of functions $f$ which lead to singularity free solutions. We require that curvature invariants must be bounded by some limiting maximal values determined by $\chi_{m}$ which is smaller than the Planck value. In this case we can ignore quantum effects and the classical nonsingluar solutions we obtain below, will be completely legitimate irrespective of quantum corrections, even close to the bounce at limiting curvatures. It is obvious that at $\chi^{2} \ll \chi_{m}^{2}$ the corrections to general relativity will be negligible only if the expansion of $f(\chi)$ at small $\chi$ starts at order $\chi^{4}$. To demonstrate 
this idea and obtain non-singluar solutions we will take the function $f(\chi)$ to be of the Born-Infeld type 1

$$
f(\chi)=1+\frac{1}{2} \chi^{2}-\chi \arcsin \chi-\sqrt{1-\chi^{2}}
$$

which after scaling $\chi \rightarrow \sqrt{\frac{2}{3}} \frac{\chi}{\chi_{m}}$ and $f \rightarrow \chi_{m}^{2} f$ takes the form

$$
f(\chi)=\chi_{m}^{2}\left(1+\frac{1}{3} \frac{\chi^{2}}{\chi_{m}^{2}}-\sqrt{\frac{2}{3}} \frac{\chi}{\chi_{m}} \arcsin \left(\sqrt{\frac{2}{3}} \frac{\chi}{\chi_{m}}\right)-\sqrt{1-\frac{2}{3} \frac{\chi^{2}}{\chi_{m}^{2}}}\right),
$$

and leads, using (19), to the particularly simple equation

$$
\chi_{m}^{2}\left(1-\sqrt{1-\frac{2}{3} \frac{\chi^{2}}{\chi_{m}^{2}}}\right)=\varepsilon
$$

We have denoted by $\varepsilon$ the terms in the right hand side of equation (19), which are independent on the time derivative of metric, that is,

$$
\varepsilon=\frac{\lambda_{k}^{i} \lambda_{i}^{k}}{8 \gamma}+\frac{C}{\sqrt{\gamma}}+T_{0}^{0}
$$

By squaring equation (22) and recalling that $\chi=\dot{\gamma} / 2 \gamma$, equation (23) can be rewritten as

$$
\frac{1}{12}\left(\frac{\dot{\gamma}}{\gamma}\right)^{2}=\varepsilon\left(1-\frac{\varepsilon}{\varepsilon_{m}}\right)
$$

where $\varepsilon_{m}=2 \chi_{m}^{2}$.

\section{Nonsingular Friedmann universe}

Consider first, the flat isotropic Friedmann universe with the metric

$$
d s^{2}=d t^{2}-a^{2}(t) \delta_{i k} d x^{i} d x^{k}
$$

In this case $\gamma=a^{6}$, and $\lambda_{k}^{i}=0$. Correspondingly, equation (24) becomes

$$
3\left(\frac{\dot{a}}{a}\right)^{2}=\frac{\varepsilon_{m}}{a^{3}}\left(1-\frac{1}{a^{3}}\right)
$$

\footnotetext{
${ }^{1}$ The use of the multivalued functions $\arcsin \chi$ and $\sqrt{1-\chi^{2}}$ could be avoided by first defining a function $\chi=\sin \psi$. We thank Alain Connes for pointing this to us.
} 
where we have normalized the scale factor $a$ in such a way as to have $\varepsilon=\varepsilon_{m}$ at $a=1$ for mimetic matter and skipped the contribution of the other matter, that is, $T_{0}^{0}=0$. This equation can be easily integrated and its solution

$$
a=\left(1+\frac{3}{4} \varepsilon_{m} t^{2}\right)^{1 / 3},
$$

describes first the contracting universe which is cold matter dominated for $t<-1 / \sqrt{\varepsilon_{m}}$, where $a \propto t^{2 / 3}$, then it passes through the regular bounce during time interval $-1 / \sqrt{\varepsilon_{m}}<t<1 / \sqrt{\varepsilon_{m}}$. Finally after the bounce for $t>$ $1 / \sqrt{\varepsilon_{m}}$ the universe is expanding as the normal dust dominated Friedmann universe with $a \propto t^{2 / 3}$. To see how the usual hydrodynamical matter will influence the bounce, if it dominates at the limiting curvature, let us neglect mimetic matter and consider the flat universe filled by matter with constant equation of state $p=w \varepsilon$. Equation (25) then becomes

$$
3\left(\frac{\dot{a}}{a}\right)^{2}=\frac{\varepsilon_{m}}{a^{3(1+w)}}\left(1-\frac{1}{a^{3(1+w)}}\right),
$$

and its solution

$$
a=\left(1+\frac{3}{4}(1+w)^{2} \varepsilon_{m} t^{2}\right)^{\frac{1}{3(1+w)}}
$$

is regular at the bounce. One can easily see that all solutions for the contracting universe with an arbitrary spacial curvature are regular near the bounce and all invariants of the curvature remain bounded. In fact, the spatial curvature term is proportional to $1 / a^{2}$ and it can obviously be neglected at high curvatures compared to the mimetic dust or usual hydrodynamical matter with equation of state $w>-1 / 3$. In the vicinity of the bounce, which happens when $\varepsilon\left(a_{m}\right)=\varepsilon_{m}$, we can approximate (24) as

$$
3\left(\frac{\dot{a}}{a}\right)^{2} \simeq-\left(\frac{d \varepsilon}{d a}\right)_{m}\left(a-a_{m}\right) .
$$

Since $\varepsilon$ grows as a decreases the derivative $d \varepsilon / d a$ is negative and hence $a$ must be always larger than or equal to $a_{m}$. The solution of the equation above, near the bounce, is given by

$$
a \simeq a_{m}+\left|\frac{a_{m}^{2}}{12}\left(\frac{d \varepsilon}{d a}\right)_{m}\right| t^{2}
$$


which remains regular irrespective of the matter content and the spatial curvature of the universe. Obviously all curvature invariants are also bounded and regular.

\section{Avoiding the singularity in Kasner universe}

Now we turn to the case of contracting Kasner universe. The Kasner Universe is the homogeneous, but anisotropic solution of Einstein's equation in empty space

$$
d s^{2}=d t^{2}-t^{2 p_{1}} d x^{2}-t^{2 p_{2}} d y^{2}-t^{2 p_{3}} d z^{2},
$$

where the constants $p_{i}$ satisfy the conditions [8]

$$
p_{1}+p_{2}+p_{3}=1, \quad p_{1}^{2}+p_{2}^{2}+p_{3}^{2}=1 .
$$

For $t<0$ this metric describes the contracting universe, while for positive $t$ an expanding homogeneous anisotropic universe. The scalar curvature and Ricci tensor square for solution (31) vanish, that is, $R=0$ and $R_{\alpha \beta} R^{\alpha \beta}=$ 0 . However, space-time is curved because the Riemann curvature squared invariant is equal to

$$
R_{\alpha \beta \gamma \delta} R^{\alpha \beta \gamma \delta}=-\frac{16}{t^{4}} p_{1} p_{2} p_{3},
$$

and becomes infinite at $t=0$, thus showing that there is a final singularity in contracting universe and initial singularity in expanding universe. We will now show how in our theory this singularity is resolved by limiting curvature, and will obtain the bouncing Kasner solution. To find this solution let us consider the metric

$$
\gamma_{i k}=\gamma_{(i)}(t) \delta_{i k}
$$

the determinant of which is equal to $\gamma=\gamma_{(1)} \gamma_{(2)} \gamma_{(3)}$. To simplify the formulae we first consider empty universe without any matter and later on will show why the presence of matter does not change the main conclusion about absence of the singularity. In empty universe, $\varepsilon$ defined in (23), is given by

$$
\varepsilon=\frac{\lambda_{k}^{i} \lambda_{i}^{k}}{8 \gamma}
$$

where $\lambda_{k}^{i}$ do not depend on time and and it is traceless, $\lambda_{i}^{i}=0$. Equation (24) then becomes

$$
\left(\frac{\dot{\gamma}}{\gamma}\right)^{2}=\frac{3 \bar{\lambda}^{2}}{2 \gamma}\left(1-\frac{\bar{\lambda}^{2}}{8 \varepsilon_{m} \gamma}\right),
$$


where we have denoted $\bar{\lambda}^{2} \equiv \lambda_{k}^{i} \lambda_{i}^{k}$. This equation can be easily integrated and the result is

$$
\gamma=\frac{\bar{\lambda}^{2}}{8 \varepsilon_{m}}\left(1+3 \varepsilon_{m} t^{2}\right)
$$

Thus, the determinant of the metric remains finite and bounded from below. We now find the components of the metric. Without loss in generality we can diagonalize $\lambda_{k}^{i}$ so that its eigenvalues are $\lambda_{(i)}=\left(\lambda_{1}, \lambda_{2}, \lambda_{3}\right)$. In this case,

$$
\varkappa_{k}^{i}=\gamma^{i m} \dot{\gamma}_{m k}=\frac{\dot{\gamma}_{(i)}}{\gamma_{(i)}} \delta_{k}^{i}
$$

and from equation (18) it follows that the components of the metric satisfy

$$
\frac{\dot{\gamma}_{(i)}}{\gamma_{(i)}}=\frac{1}{3} \frac{\dot{\gamma}}{\gamma}+\frac{\lambda_{(i)}}{\sqrt{\gamma}}
$$

Since $\gamma$ is bounded from below and $\dot{\gamma} / \gamma$ always remains analytical and finite near the bounce all curvature invariants built out of $\varkappa_{k}^{i}$ and its derivatives are non-singluar. Integrating equation (38) we obtain

$$
\gamma_{(i)}=\gamma^{1 / 3} \exp \left(\lambda_{(i)} \int \frac{d t}{\sqrt{\gamma}}\right)
$$

and substituting for $\gamma$ from (36) we find

$$
\gamma_{(i)}=\left(\frac{\bar{\lambda}^{2}}{8 \varepsilon_{m}}\left(1+3 \varepsilon_{m} t^{2}\right)\right)^{1 / 3} \exp \left(2 \sqrt{\frac{2}{3}} \frac{\lambda_{(i)}}{\bar{\lambda}} \sinh ^{-1}\left(\sqrt{3 \varepsilon_{m}} t\right)\right)
$$

Taking into account that for $\varepsilon_{m} t^{2} \gg 1$

$$
\sinh ^{-1}\left(\sqrt{3 \varepsilon_{m}} t\right) \simeq \pm \ln \left|2 \sqrt{3 \varepsilon_{m}} t\right|
$$

where the plus and minus signs are to be taken for $t \gg 1 / \sqrt{\varepsilon_{m}}$ and $t \ll$ $-1 / \sqrt{\varepsilon_{m}}$, respectively, then this metric simplifies to

$$
\gamma_{(i)} \simeq\left(\frac{\bar{\lambda}^{2}}{32 \varepsilon_{m}}\right)^{1 / 3}\left(12 \varepsilon_{m} t^{2}\right)^{p_{i}^{ \pm}}
$$

where

$$
p_{i}^{ \pm}=\frac{1}{3} \pm \sqrt{\frac{2}{3}} \frac{\lambda_{(i)}}{\bar{\lambda}}
$$


Since $\lambda_{1}+\lambda_{2}+\lambda_{3}=0$, the $p_{i}^{ \pm}$satisfy the conditions

$$
p_{1}^{ \pm}+p_{2}^{ \pm}+p_{3}^{ \pm}=1, \quad\left(p_{1}^{ \pm}\right)^{2}+\left(p_{2}^{ \pm}\right)^{2}+\left(p_{3}^{ \pm}\right)^{2}=1,
$$

and we have the familiar Kasner solution (31) at low curvatures. Only when the curvature becomes of order $\varepsilon_{m}$, the Kasner contraction changes and for $\varepsilon_{m} t^{2} \ll 1$ the metric

$$
\gamma_{(i)} \simeq\left(\frac{\bar{\lambda}^{2}}{8 \varepsilon_{m}}\right)^{1 / 3}\left(1+\frac{\lambda_{(i)}}{\bar{\lambda}} \sqrt{8 \varepsilon_{m}} t\right)
$$

describes the regular bounce and finally an expanding Kasner universe. Notice that during the bounce the indices characterizing the Kasner universe are changing. If in a contracting universe they were $p_{i}^{-}$then after the bounce they become

$$
p_{i}^{+}=\frac{2}{3}-p_{i}^{-} .
$$

In particular, the Kasner Universe with $p_{i}^{-}=\left(-\frac{1}{3}, \frac{2}{3}, \frac{2}{3}\right)$ turns after the bounce to the Kasner universe with $p_{i}^{+}=(1,0,0)$ for which all curvature invariants, including the Riemann tensor squared given in (33), are equal to zero. Hence after the bounce the Kasner universe evolves to Minkowski space-time. In fact, the coordinate transformation

$$
T=t \cosh x, \quad X=t \sinh x
$$

brings the Kasner metric

$$
d s^{2}=d t^{2}-t^{2} d x^{2}-d y^{2}-d z^{2},
$$

to the standard Minkowski form

$$
d s^{2}=d T^{2}-d X^{2}-d y^{2}-d z^{2} .
$$

The curvature invariants for the non-singluar solution (40) are bounded and regular everywhere. In fact, substituting (36) into the expressions

$$
\begin{gathered}
R=-\dot{\varkappa}-\frac{1}{3} \varkappa^{2}-\frac{\bar{\lambda}^{2}}{4 \gamma}, \\
R_{\alpha \beta} R^{\alpha \beta}=\frac{1}{3} \dot{\varkappa}^{2}+\frac{1}{6} \varkappa^{2} \dot{\varkappa}+\frac{1}{36} \varkappa^{4}+\frac{1}{4 \gamma}\left(\dot{\varkappa}+\frac{1}{6} \varkappa^{2}\right) \bar{\lambda}^{2}+\frac{1}{16 \gamma^{2}} \bar{\lambda}^{4}
\end{gathered}
$$




$$
R_{\alpha \beta \gamma \delta} R^{\alpha \beta \gamma \delta}=\left(\frac{\varkappa^{4}}{54}+\frac{\dot{\varkappa}^{2}}{3}+\frac{\varkappa^{2} \dot{\varkappa}}{9}\right)+\frac{\left(4 \dot{\varkappa}+\varkappa^{2}\right)}{12 \gamma} \bar{\lambda}^{2}-\frac{\varkappa}{\gamma^{\frac{3}{2}}} \lambda_{1} \lambda_{2} \lambda_{3}+\frac{3}{16 \gamma^{2}} \bar{\lambda}^{4}
$$

which are quoted above, for convenience of the reader, we find

$$
R=-\frac{8 \varepsilon_{m}}{\left(1+3 \varepsilon_{m} t^{2}\right)^{2}}, \quad R_{\alpha \beta} R^{\alpha \beta}=\frac{28 \varepsilon_{m}^{2}}{\left(3 \varepsilon_{m} t^{2}+1\right)^{4}},
$$

and

$R_{\alpha \beta \gamma \delta} R^{\alpha \beta \gamma \delta}=\frac{8 \varepsilon_{m}^{2}\left(12 t^{4} \varepsilon_{m}^{2}+6 t^{2} \varepsilon_{m}+5\right)}{\left(3 \varepsilon_{m} t^{2}+1\right)^{4}}-\frac{16}{t^{4}}\left(\frac{3 \varepsilon_{m} t^{2}}{1+3 \varepsilon_{m} t^{2}}\right)^{5 / 2}\left(p_{1} p_{2} p_{3}+\frac{2}{27}\right)$

Hence, not only the invariants but also all their derivatives are finite at any moment of time. For $\varepsilon_{m} t^{2} \gg 1$ the solution approaches the Kasner solution in the leading order, up to higher order corrections. For instance, at large $t$ the scalar curvature is not exactly vanishing and it is of order

$$
R \sim \frac{1}{\varepsilon_{m}} R_{\alpha \beta \gamma \delta} R^{\alpha \beta \gamma \delta}
$$

which in $\varepsilon_{m}^{-1}$ times exceeds the vacuum polarization corrections, and at low curvatures are negligible, unless $\varepsilon_{m}$ is not much smaller than the Planckian value.

Finally we discuss how the presence of matter can influence the behavior of Kasner solutions in the vicinity of a bounce. It is clear that if at some moment in the past at $t_{0}<-\varepsilon_{m}^{-1 / 2}$ the curvature contribution in (23) dominates over the the usual matter, that is,

$$
\frac{\bar{\lambda}^{2}}{\gamma\left(t_{0}\right)}>T_{0}^{0}\left(t_{0}\right)
$$

then $T_{0}^{0}(t)$ in (23) can be neglected if the matter equation of state satisfies $w<1$. In fact, $T_{0}^{0} \propto \gamma^{-\frac{1+w}{2}}$ does not grow as fast as $\bar{\lambda}^{2} / \gamma$ if $\gamma$ decreases, and therefore can be neglected when we approach the bounce. On the other hand when $w>1$ the matter term can start to dominate well before the bounce, and in this case the bounce happens as in the Friedmann universe considered above. 
If matter (for instance, the scalar field) changes the equation of state from $w \gg 1$ before the bounce to $w<1$ after the bounce, the Kasner universe which was strongly anisotropic can create homogeneous isotropic Friedmann universe after the bounce. In fact, at large $\gamma$ in contracting Kasner universe, the usual matter with $w \gg 1$ can be completely ignored because $T_{0}^{0} \propto \gamma^{-\frac{1+w}{2}}$ and this matter becomes relevant only at small $\gamma$ close to the bounce. If the equation of state during or after the bounce changes to $w<1$ then during the expansion stage, $T_{0}^{0}$ will be decaying not as fast as $\bar{\lambda}^{2} / \gamma$, and finally begins to dominate. In this case $\dot{\gamma} / \gamma \propto \gamma^{-\frac{1+w}{4}}$ and the second term in (38), which spoils the isotropy and decay as $\lambda_{i} / \sqrt{\gamma}$ will finally become negligible compared to $\dot{\gamma} / \gamma$. As a result the universe will become isotropic. This opens the possibility to solve the isotropy problem in bouncing cosmology.

\section{Discussion}

We have shown above that the singularities in cosmological solutions can be easily removed by making the longitudinal degree of freedom of gravity to be dynamical. The presence of mimetic field needed to generate Born-Infeld corrections to the Einstein theory adds extra "dust like" degree of freedom to gravity, which can serve as mimetic dark matter. It is rather curious that the non-singluar modification of General Relativity delivers for free the realistic candidate for Dark Matter. Besides of the appearance of mimetic matter, Einstein's equations are significantly modified only at very high curvatures which are close to the limiting one.

In the considerations above we have chosen a particular function $f(\square \phi)$ to get the simplest possible equations. One can wonder to what extent this choice is unique? Our preliminary investigation shows that there is a whole class of functions $f$ which would lead to theories with limiting curvature. However this class is rather restricted. In particular, the necessary ingredients common to all these functions are 1) they have to contain a Born-Infeld type term, and 2) the derivative $\frac{d f}{d \chi}$ must remain finite when $\chi$ approaches its limiting value $\chi_{m}$.

The non-singluar models we have constructed can be rather important for bouncing cosmologies. In fact, in these models a bounce happens within very short time interval of order $t \sim \varepsilon_{m}^{-1 / 2}$ and if the limiting curvature would be of the order of Planck value, this time would be the Planckian time. Outside this time interval Einstein's theory is well applicable. Using causality 
one can argue that in this case perturbations generated in a contracting universe on the supercurvature scale can be re-translated to an expanding universe practically without any change. However, this question requires further quantitative investigation.

The idea of limiting curvature can also have rather severe consequences for inflationary universe. First of all, notice that if we want to use the stage of accelerated expansion for amplifying quantum fluctuations, observed in numerous CMB experiments, the limiting curvature cannot be below the inflationary scale, that is, it cannot be less than the Planckian scale more than just by few orders of magnitude. When the limiting curvature is below the self-reproduction scale the multiverse is avoided. The flat inflationary potentials, favoured by observations, also look more natural from the point of view of the idea of limiting curvature.

The theory we have considered here is a classical theory and if the limiting curvature is well below the Planck scale we can safely ignore quantum corrections. However, any field gravitates, and therefore the highly energetic quanta which in Einstein's theory would produce the curvature exceeding the limiting one, must be either prohibited or modified. This is why we expect that the natural cut-off in quantum field theories, which is normally taken to be of the order of Planck value, can be well below the Planck scale in theories with limiting curvature.

The considerations above are restricted to highly symmetric space-times. One could naturally address the question whether the curvature will remain bounded generically for arbitrary inhomogeneous spaces. In an accompanying paper [12] we show that the singularity is also avoided in the case of a black hole and give the explicit solution for it. Moreover analyzing the expressions for the curvature invariants one could argue that the limiting curvature is the generic property of arbitrary space-time irrespective of their spatial curvature. However these questions require a more serious investigation.

\section{Acknowledgments}

The work of A. H. C is supported in part by the National Science Foundation Grant No. Phys-1518371. The work of V.M. is supported in part by Simons Foundation grant 403033TRR 33 and "The Dark Universe" and the Cluster of Excellence EXC 153 "Origin and Structure of the Universe". 


\section{References}

[1] S. W. Hawking and R. Penrose, The singularities of gravitational collapse and cosmology, Proc. Roy. Soc. Lond. A. 314 (1970) 529.

[2] G. W. Gibbons and S. W. Hawking, Cosmolgical event horizons, thermodynamics and particle creation, Phys. Rev. D 15 (1977) 2738.

[3] M. Markov, Pis'ma Zh. Eskp. Teor. Fiz. 36 (1982) 214; 46 (1987) 341 [JETP Lett. 36 (1982) 265; 46 (1987) 431.

[4] V. Mukhanov and R. Brandenberger, A Nonsingular Universe, Phys. Rev. Lett. 68 (1992) 1969.

[5] V. Mukhanov, R. Brandenberger, and A. Sornborger, A Cosmological Theory without Singularities, Phys. Rev. D 48 (1993) 1629.

[6] A. H. Chamseddine and V. Mukhanov, Mimetic Dark Matter, JHEP 1311 (2013) 135.

[7] A. H. Chamseddine, V. Mukhanov and A. Vikman, Cosmology with Mimetic Matter, JCAP 1406 (2014) 017.

[8] L. Landau and E. Lifshitz, The Classical Theory of Fields fourth edition, Butterworth, Heinemann, 1980.

[9] A. H. Chamseddine, A. Connes and V. Mukhanov, Quanta of Geometry: Noncommutative Aspects, Phys. Rev. Lett. 114 (2015) 091302.

[10] A. H. Chamseddine and A. Connes, Scale Invariance in the Spectral Action, J. Math. Phys. 47 (2006) 063504.

[11] A. H. Chamseddine and A. Connes, The Uncanny Precision of the Spectral Action, Comm. Math. Phys. 293 (2010) 867.

[12] A. H. Chamseddine, V. Mukhanov, Resolving Cosmological Singularities. 